\title{
Preventing Depression among Early Adolescents in the Primary Care Setting: Erratum
}

\author{
Jane E. Gillham • John Hamilton • Derek R. Freres • \\ Ken Patton • Robert Gallop
}

Published online: 10 January 2008

(C) Springer Science + Business Media, LLC 2007

A paragraph describing intervention effects on explanatory style for negative events was accidentally left out of the paper Gillham, J.E., Hamilton, J., Freres, D.R., Patton, K.,

The online version of the original article can be found at http://dx.doi. org/10.1007/s10802-005-9014-7.

J. E. Gillham $(\bowtie)$

Psychology Department, Swarthmore College,

500 College Avenue,

Swarthmore, PA 19081, USA

e-mail: jgillha1@swarthmore.edu

J. E. Gillham

Psychology Department, University of Pennsylvania,

Philadelphia, PA, USA

e-mail: jgillham@psych.upenn.edu

J. Hamilton $\cdot$ K. Patton

The Permanente Medical Group of California,

San Diego, CA, USA

J. Hamilton

Kellogg College, University of Oxford,

Oxford, UK

D. R. Freres

Annenberg School for Communication,

University of Pennsylvania,

Philadelphia, PA, USA

R. Gallop

Department of Mathematics Applied Statistics Program,

West Chester University,

West Chester, USA
\& Gallop, R. (2006). Preventing Depression among Early Adolescents in the Primary Care Setting: A randomized controlled study of the Penn Resiliency Program, Journal of Abnormal Child Psychology, 34, 203-219. The missing paragraph is presented below along with Table 2 from the original paper which presents mean scores for explanatory style for negative events $(\mathrm{CN})$ on the Children's Attributional Style Questionnaire (CASQ: Seligman et al. 1984).

\section{Explanatory Style for Negative Events}

The Penn Resiliency Program (PRP) tended to improve explanatory style for negative events, $F(1,216.21)=2.85$, $p<0.10, \mathrm{ES}=-0.16$ (95\% CI: -0.35 to 0.03$)$. Further analyses revealed a significant sex by condition interaction, $F(1,212.80)=4.57, p<0.05$. PRP significantly improved explanatory style for negative events in girls, $F(1$, 108.14) $=9.49, \quad p<0.01, \mathrm{ES}=-0.40(95 \% \mathrm{CI}:-0.65$ to -0.14) but not in boys (see Table 2). PRP's effect on explanatory style for negative events was not significantly moderated by symptom level.

\section{References}

Seligman, M. E. P., Peterson, C., Kaslow, N. J., Tanenbaum, R. L., Alloy, L. B., \& Abramson, L. Y. (1984). Explanatory style and depressive symptoms among school children. Journal of Abnormal Psychology, 93, 235-238. 
Table 2 Mean explanatory style for negative events $(\mathrm{CN})$ scores by condition: raw mean (SD)

\begin{tabular}{|c|c|c|c|c|c|c|}
\hline & \multicolumn{2}{|c|}{ All Participants } & \multicolumn{2}{|l|}{ Girls } & \multicolumn{2}{|l|}{ Boys } \\
\hline & Prevention & Usual Care & Prevention & Usual Care & Prevention & Usual Care \\
\hline \multirow[t]{2}{*}{ Baseline } & $7.85(2.95)$ & $8.02(3.25)$ & $7.52(2.90)$ & $8.20(3.13)$ & $8.22(2.98)$ & $7.83(3.40)$ \\
\hline & $\mathrm{n}=142$ & $\mathrm{n}=112$ & $\mathrm{n}=74$ & $\mathrm{n}=57$ & $\mathrm{n}=68$ & $\mathrm{n}=55$ \\
\hline \multirow[t]{2}{*}{ Post } & $7.57(3.25)$ & $8.13(3.11)$ & $6.68(2.93)$ & $8.42(2.90)$ & $8.69(3.30)$ & $7.76(3.36)$ \\
\hline & $\mathrm{n}=111$ & $\mathrm{n}=104$ & $\mathrm{n}=62$ & $\mathrm{n}=59$ & $\mathrm{n}=49$ & $\mathrm{n}=45$ \\
\hline \multirow[t]{2}{*}{6 months } & $7.40(3.26)$ & $8.32(3.04)$ & $6.60(3.03)$ & $8.44(2.67)$ & $8.33(3.31)$ & $8.16(3.53)$ \\
\hline & $\mathrm{n}=110$ & $\mathrm{n}=100$ & $\mathrm{n}=59$ & $\mathrm{n}=59$ & $\mathrm{n}=51$ & $\mathrm{n}=41$ \\
\hline \multirow[t]{2}{*}{12 months } & $7.56(3.52)$ & $8.30(3.36)$ & $7.15(3.26)$ & $8.35(3.12)$ & $8.03(3.79)$ & $8.22(3.78)$ \\
\hline & $\mathrm{n}=101$ & $\mathrm{n}=90$ & $\mathrm{n}=54$ & $\mathrm{n}=57$ & $\mathrm{n}=47$ & $\mathrm{n}=33$ \\
\hline \multirow[t]{2}{*}{18 months } & $7.83(3.38)$ & $8.14(3.29)$ & $7.20(3.12)$ & $7.62(2.74)$ & $8.84(3.57)$ & 9.03 (3.96) \\
\hline & $\mathrm{n}=96$ & $\mathrm{n}=82$ & $\mathrm{n}=59$ & $\mathrm{n}=52$ & $\mathrm{n}=37$ & $\mathrm{n}=30$ \\
\hline \multirow[t]{2}{*}{24 months } & $8.60(3.75)$ & $8.96(3.22)$ & $7.58(3.51)$ & $8.59(2.88)$ & $9.98(3.67)$ & $9.54(3.66)$ \\
\hline & $\mathrm{n}=96$ & $\mathrm{n}=82$ & $\mathrm{n}=55$ & $\mathrm{n}=50$ & $\mathrm{n}=41$ & $\mathrm{n}=32$ \\
\hline
\end{tabular}

